\title{
SUBTYPING OF CHILEAN METHICILLIN-RESISTANT STAPHYLOCOCCUS AUREUS STRAINS CARRYING THE STAPHYLOCOCCAL CASSETTE CHROMOSOME MEC TYPE I
}

\author{
Gustavo Medina ${ }^{1}$, Carola Otth', Laura Otth¹, Heriberto Fernández ${ }^{1}$, Celeste Muñoz ${ }^{1}$, María Cruz $^{2}$, Ángela Zaror $^{2}$, \\ Ruby Henriquez ${ }^{2}$, Maria Arce², Myra Wilson ${ }^{1 *}$ \\ ${ }^{1}$ Instituto de Microbiología Clínica, Facultad de Medicina, Universidad Austral de Chile, Valdivia, Chile; ${ }^{2}$ Sección \\ Microbiología, Laboratorio Central. Hospital Clínico Regional Valdivia.
}

\begin{abstract}
The cassette chromosome mec (SCCmec) present in methicillin-resistant Staphylococcus aureus (MRSA) has two essential components, the $c c r$ gene complex and the mec gene complex. Additionally, SCCmec has non-essential components called $\mathrm{J}$ regions which are used for MRSA subtyping. This study was performed to determine subtypes MRSA strains carrying SCCmec type I based on polymorphism of regions located downstream of the mecA gene. A total of 98 MRSA strains carrying SCCmec type I isolated from patients hospitalized at the County Hospital of Valdivia (Chile) between May 2007 and May 2008, were analyzed by multiplex PCR designed to amplify the mecA gene and 7 DNA hypervariable regions located around the $m e c A$ gene. MRSA strains were classified into seventeen genotypes accordingly to amplification patterns of DNA hypervariable regions. Five genotypes showed amplification patterns previously described. The remaining twelve genotypes showed new amplification patterns. Genotypes 18 and Genotype 19 were the most frequently detected. Regions HVR, Ins117 and pI258 stand out as being present in more than $60 \%$ of tested isolates. The acquisition of hypervariable regions by MRSA is a continuous horizontal transfer process through which the SCCmec have been preserved intact, or even may give rise to new types and subtypes of SCCmec. Therefore it is possible to infer that most MRSA strains isolated at the County Hospital of Valdivia (Chile) were originated from two local clones which correspond to Genotype 18 and Genotype 19.
\end{abstract}

Key words: Subtypified MRSA, polymorphism MRSA, SCCmec MRSA.

\section{INTRODUCTION}

Methicillin-resistant Staphylococcus aureus (MRSA) was first isolated in England in 1961 shortly after the development of methicillin (8). Since then, MRSA has become the most prevalent pathogen causing hospital infection throughout the world, with increased incidence in many countries (2).

MRSA genome has integrated a mobile genetic

\footnotetext{
*Corresponding Author. Mailing address: Instituto de Microbiologia Clinica, Facultad de Medicina, Universidad Austral de Chile. P.O. Box: 567, Valdivia, Chile.; Tel.: 5663221921 Fax: 5663 293300.; E-mail: myrawilson@uach.cl
} 
element called staphylococcal cassette chromosome mec (SCCmec), which harbors the mecA gene responsible for methicillin resistance. This gene encodes PBP2a, an additional ßlactam-resistant penicillin-binding protein (4). SCCmec is a unique genomic island found only in staphylococcal species that have two essential components, the $c c r$ gene complex ( $c c r)$ and the mec gene complex (mec) $(2,5)$. The ccr gene complex is composed of $c c r$ genes and surrounding open reading frames (ORFs). The mec gene complex is composed of the mecA gene, regulatory genes, and insertion sequences upstream or downstream of $m e c A$ gene $(6,7)$.

Remaining parts of SCCmec are called J regions (J1, J2 and J3), which constitute nonessential components of SCCmec. In some cases, these regions carry additional antibiotic resistance determinants (5). $\mathrm{J} 1$ is the region between the chromosomal left junction and the $c c r$ complex; $\mathrm{J} 2$ is the region between the $c c r$ complex and the mec complex and $\mathrm{J} 3$ is the region between the mec complex and the chromosomal right junction. Variations in the $J$ regions are used for subtyping MRSA strains (9).

Currently, different genetic methods have been developed to be applied in molecular epidemiologic characterization of MRSA strains, being pulsed-field electrophoresis (PFGE) the technique of choice (14). On the other hand, through multiplex PCR technique it is possible to analyze the polymorphic downstream of mecA gene. This genetic polymorphism has been used as an epidemiological marker and has also been the basis of studies related to the evolutionary origin and subtyping of methicillin resistance in $S$. aureus (3).

The aim of this study was to determine subtypes of MRSA strains carrying SCCmec type I, through the implementation of a multiplex PCR that allows the detection of mecA gene and 7 DNA hypervariable regions located around the mecA gene.

\section{MATERIALS AND METHODS}

\section{Clinical isolates}

Ninety eight clinical isolates of MRSA previously typified as SCCmec type I and unrelated to nosocomial outbreaks were studied. All of them were isolated from patients hospitalized at the County Hospital of Valdivia (Chile) between May 2007 and May 2008. Strains phenotyping was performed using the semi-automated microbiological diagnosis system Dried Gram Positive ID Type 2 panels (Microscan $^{\circledR}$ ) and SCCmec genotyping was performed as described previously (17). The mecA-positive $S$. aureus ATCC 49476, which contains HVR, pT181, pI258, mecR1 and IS256 regions was used as control.

DNA hypervariable regions Subtyping: A single colony was taken from a Muller Hinton agar plate and suspended in $100 \mu \mathrm{L}$ of sterile nuclease free water. The suspension was incubated at $100^{\circ} \mathrm{C}$ for $10 \mathrm{~min}$ for DNA extraction. After centrifugation at $20,000 \mathrm{~g}$ for $2 \mathrm{~min}, 3 \mu \mathrm{L}$ of the supernatant was taken and directly added to $25 \mu \mathrm{L}$ of amplification mixture.

Oligonucleotides sequences used for the amplification of mecA gene and 7 DNA hypervariable regions are listed in Table $1(3,17)$.

The amplification protocols originally described by Huygens et al., and Wilson et al., were modified due to the similar size of PCR amplicon $(3,15)$. The analysis of each strain was performed in four individual reactions. i) The first reaction included primers to amplify mecA gene, pI258 (I) and mecR1 regions. ii) The second reaction included primers to amplify pI258 (II) and IS256 regions. iii) The third reaction included primers to amplify pUB110 and pT181 regions. iv) The fourth reaction included primers to amplify HVR and Ins117 regions.

The PCR mixture consisted of $3 \mu \mathrm{L}$ of cell lysate, 0.2 $\mathrm{mM}$ concentrations of each deoxynucleoside triphosphate (dNTPs), $0.5 \mu \mathrm{M}$ concentrations of each primer, 1 Uof DFS Taq DNA polymerase (Invitrogen ${ }^{\circledR}$ ), 10X PCR buffer and $1.5 \mathrm{mM} \mathrm{MgCl} 2$ contained in a total volume reaction of 25 $\mu \mathrm{L}$. The program DNA amplification consisted of an initial cycle of $95^{\circ} \mathrm{C}$ for $5 \mathrm{~min}$, followed by 30 cycles of $95^{\circ} \mathrm{C}$ for $30 \mathrm{~s}, 50^{\circ} \mathrm{C}$ for $30 \mathrm{~s}$, and $72^{\circ} \mathrm{C}$ for $30 \mathrm{~s}$, with a final extension step of $72^{\circ} \mathrm{C}$ for $10 \mathrm{~min}$. PCR products were visualized on $1.5 \%$ agarose gels stained with ethidium bromide. 
Table 1. Primers used for subtyping MRSA. The analysis of each strain was performed in four individual reactions.

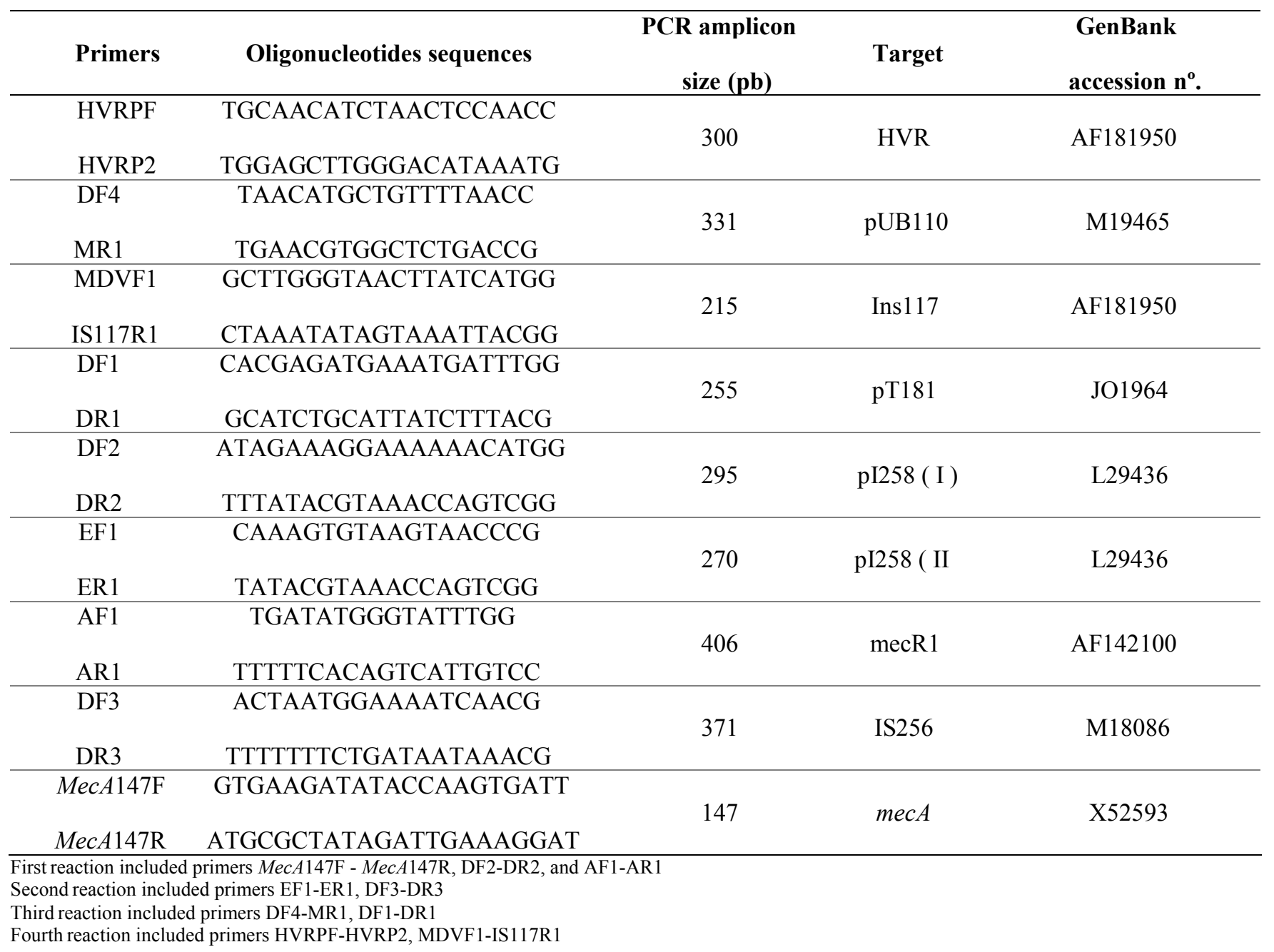

\section{RESULTS}

The present study showed that all MRSA strains, previously typified as SCCmec type I, were classified into seventeen genotypes according to amplification patterns of DNA hypervariable regions (Table 2).

Genotypes 2, 6, 14, 15 and 16 showed amplification patterns previously described by Huygens et al. and Wilson et al. A serial number, starting with the genotype 18, was assigned to the remaining twelve new amplification patterns (Table 2).
The most frequent amplification patterns found were genotypes 18 and genotype 19 with 24,5\% and 20,4\% respectively (Figure 1 and Table 2). On the other hand, five strains were classified into genotype 29 which did not detect any of the DNA hypervariable regions (Table 2).

Finally, the detection percentage of DNA hypervariable regions was: HVR 92,9\% - Ins117 and pI258 69,4\% - IS256 46,9\% - pT181 13,3\% - pUB110 2\%. In addition, we found that no strains included in the analysis amplified the mecR1 region. 
Table 2. Classification of genotypes according to amplification patterns of DNA hypervariable regions and their frequency.

\begin{tabular}{|c|c|c|c|c|c|c|c|c|}
\hline \multirow[b]{2}{*}{ Genotype } & \multicolumn{7}{|c|}{ DNA hypervariable regions } & \multirow[b]{2}{*}{ Frequency $\%$} \\
\hline & HVR & pUB110 & Ins117 & pT181 & pI258 & mecRI & IS256 & \\
\hline 2 & + & - & + & - & - & - & - & 10.2 \\
\hline 6 & + & - & - & - & - & - & - & 4.0 \\
\hline 14 & + & - & & + & + & - & - & 2.0 \\
\hline 15 & + & - & - & - & + & - & - & 8.2 \\
\hline 16 & + & - & - & - & + & - & + & 4.0 \\
\hline 18 & + & - & + & - & + & - & + & 24.5 \\
\hline 19 & + & - & + & - & + & - & - & 20.4 \\
\hline 20 & + & - & + & + & + & - & + & 6.1 \\
\hline 21 & + & - & - & - & - & - & + & 5.1 \\
\hline 22 & + & - & + & + & - & - & + & 3.0 \\
\hline 23 & + & - & + & - & - & - & + & 2.0 \\
\hline 24 & - & - & - & - & + & - & + & 1.0 \\
\hline 25 & - & + & + & - & - & - & - & 1.0 \\
\hline 26 & + & + & + & - & + & - & - & 1.0 \\
\hline 27 & + & - & - & + & + & - & + & 1.0 \\
\hline 28 & + & - & + & + & + & - & - & 1.0 \\
\hline 29 & - & - & - & - & - & - & - & 5.0 \\
\hline
\end{tabular}

98 MRSA strains isolated from patients hospitalized at the County Hospital of Valdivia (Chile), previously typified as SCCmec type I, were subtypified into seventeen genotypes according to amplification patterns of 7 DNA hypervariable regions. The genotypes 2 , 6, 14, 15 and 16 showed amplification patterns previously described. A serial number, starting with the genotype 18, was assigned to the remaining twelve new amplification patterns. Amplification patterns corresponding to genotypes 18 and genotype 19 were the most frequent.

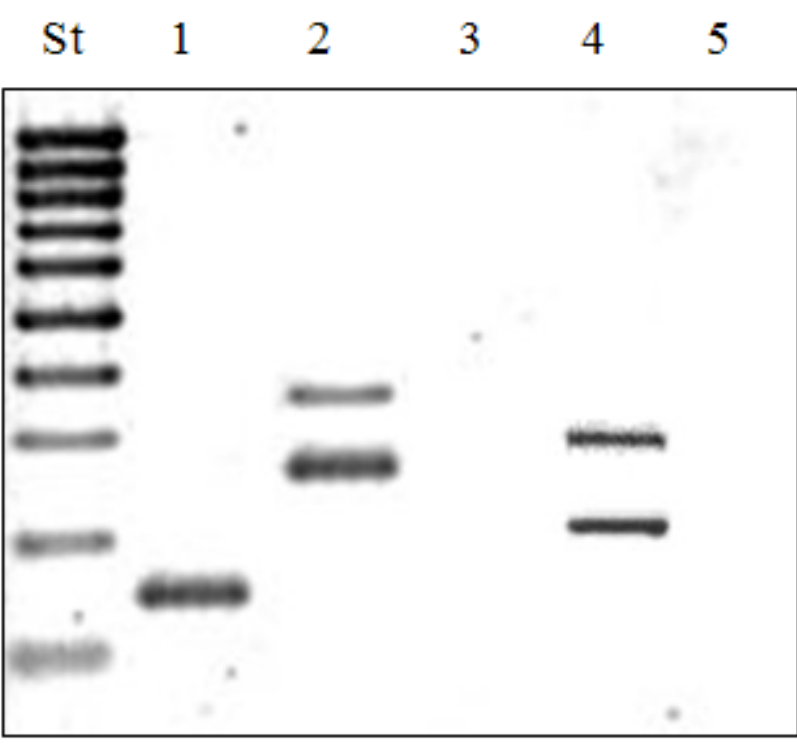

Genotype 18

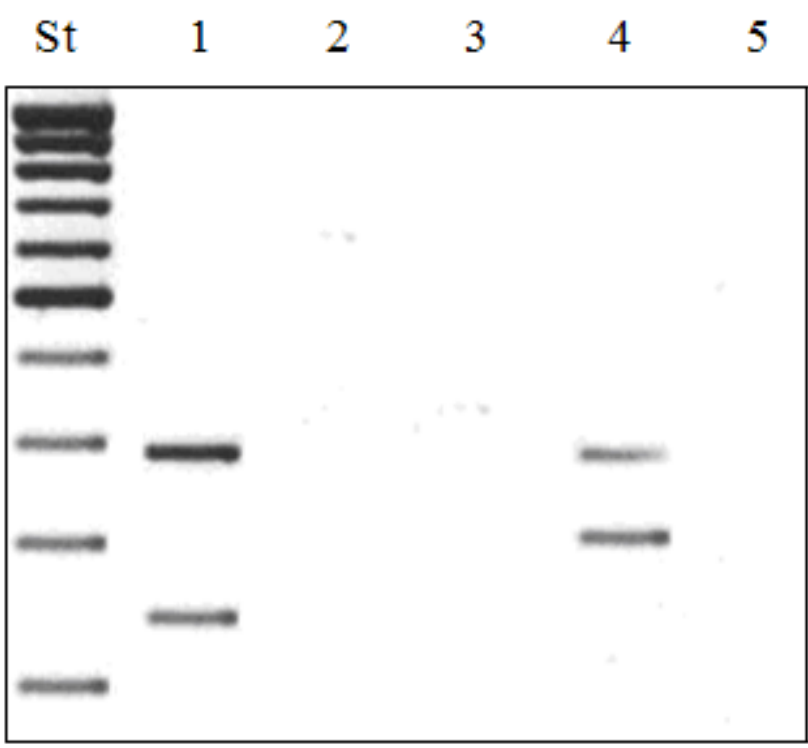

Genotype 19

Figure 1. Amplification patterns of genotype 18 and genotype 19. The most frequent amplification patterns. Genotype $18=\mathrm{St}$ : standard molecular size, 1: mecA, 2: pI258 - IS256, 4: Ins117 - HVR, 5: negative control. Genotype 19= St: standard molecular size, 1: mecA - pI258, 4: Ins117 - HVR, 5: negative control. 


\section{DISCUSSION}

SCCmec typing is one of the most important molecular tools available for understanding the epidemiology and clonal strain relatedness of MRSA (14). However, due to the very complex and diverse structure of the SCCmec element, SCCmec subtyping is a powerful tool applicable to clinical and epidemiological surveillance purposes (10). Based on the horizontal transfer of SCCmec and the polymorphism of regions located "downstream" of the mecA gene, we suggest that genotypes identified through the presence of hypervariable regions can be classified as subtypes of MRSA strains previously typified as $\mathrm{SCCmec}$ type I.

In the present study ninety eight MRSA strains isolated from patients hospitalized at the County Hospital of Valdivia (Chile), were subtypified into seventeen genotypes according to amplification patterns of 7 DNA hypervariable regions located around the mec $A$ gene.

Seventeen genotypes detected in our environment contrasts with the five genotypes previously identified by Wilson et al., who detected only five genotypes of MRSA strains isolated from patients hospitalized at the County Hospital of Valdivia (Chile) between March 2004 and December 2005 (15). This situation is because in our study we included a greater number of strains and we identified hypervariable regions not detected previously.

The new amplification patterns detected in this study were ranked between genotype 18 and genotype 29. There was a predominance of genotype 18 and genotype 19 with $24.5 \%$ and 20,4\% respectively (Figure 1 and Table 2). From these data we could infer that most of MRSA strains were originated from two local clones. In fact, we suggest that strains belonging to genotype 18 are different from those belonging to genotype 19, does not possess the IS256 region. Therefore, we infer that the strains belonging to genotype 18 come from a strain belonging to genotype 19, in which the IS256 region was integrated.

IS256 region, located downstream of a fragment $2 \mathrm{~Kb}$ called $d c s$ (downstream constant segment), is an insertion sequence that can be independent or as part of the transposon Tn4001. This transposon carries the aacA-aphD gene, which encodes resistance to aminoglycoside $(1,11)$. IS256 region was detected in $46.9 \%$ of MRSA strains. These results are different from those obtained by Wilson et al., who detected this region in $9.4 \%$ of MRSA strains (15).

The increase in the prevalence of IS256 region is probably due to a clonal expansion of some MRSA strains that possess this region in their SCCmec.

Moreover, this situation reflects the constant genomics evolution of MRSA strains in our environment. In two years (2005 - 2007), almost half of strains incorporated the IS256 region in their SCCmec. This is worrying because IS256 region allows the insertion of $\operatorname{Tn} 4001$ encoding resistance to aminoglycoside (11).

Ins117 region is a short sequence of $117 \mathrm{bp}$, flanked by two $15 \mathrm{bp}$ direct repeats, contained within $\operatorname{orf} X$ region (11). This region was detected in $69.4 \%$ of MRSA strains. These results are different from those obtained by Wilson et al., who did not detect this region in MRSA strains (15). The increase in the prevalence of Ins 117 region is probably due to a clonal expansion of some MRSA strain that possess this region in their SCCmec as happened with IS256 region. This is also worrying because Ins117 region, along with IS431, allows the insertion of plasmid pUB110 which encodes resistance to tetracycline and aminoglycoside $(11,12)$.

pUB110 region is flanked by IS431 and was integrated during the period when mec DNA was being formed and prior to the emergence of the first outbreaks of MRSA infections in European hospitals in the early 1960 s $(11,12)$. This region was detected in $2 \%$ of MRSA strains. In the previous study of Wilson et al. MRSA strains carrying pUB110 region were not detected (15). Spread of strains possessing pUB110 region would be a problem due to the resistance that this region encodes. Moreover, pUB110 region is present in subtypes SCCmec IA, II-A, II-b, II-A, II-B and II-C. MRSA strains showed this region can be classified as SCCmec subtype IA (16).

HVR region is a DNA sequence composed by direct 
repeat unit elements (DRUs) located between IS431mec and mecA (13). This region was detected in $92.9 \%$ of MRSA strains. A similar situation is reported by Wilson et al (15). This fact reflects the high degree of conservation of the HVR region at the strains isolated in our hospital environment.

pI258 and pT181 regions are plasmid flanked by IS431 that encodes resistance to mercury and tetracycline respectively (11). pI258 region was detected in $69.4 \%$ of our strains. Previously Wilson et al. detected the $\mathrm{pI} 258$ region in $81 \%$ of their MRSA strains (15). pT181 region was detected in $13.3 \%$ of our strains. These results are different from those obtained by Wilson et al. who detected this region in $41.5 \%$ of their MRSA strains (15).

Located upstream of mecA gene lies the mecR 1 gene, that encodes the protein MecR1, which activates the mecA gene transduction generating the synthesis of PBP2a $(2,16)$. This region was not detected in any of our MRSA strains, as it was previously reported by Wilson et al (15). This situation is because mecR1 gene is characterized by suffering deletions. This characteristic is highly conserved among strains isolated in our environment (2).

Finally, based on the results obtained in this study and the results obtained previously by Wilson et al (15) we suggest that acquisition of hypervariable regions by MRSA is a continuous horizontal transfer process through which the SCCmec has been preserved intact, or even may give rise to new types and subtypes of SCCmec. This means that MRSA strains could maintain or increase their resistance, but in no case it would decrease.

Continue surveillance studies are needed to make annual checkups to determine the prevalence of MRSA subtypes in our environment, as well as controlling the emergence of new subtypes. On the other hand, it would allow retrospective studies to detect evolutionary changes and would establish an accurate antimicrobial therapy, which would shorten the hospitalization stay, resulting in a significant decrease in health costs caused by MRSA infections.

\section{ACKNOWLEDGEMENTS}

This work was supported by Direction of Research and Development of the Universidad Austral de Chile (DID-UACh-
S-2007-63 and S-2010-02).

\section{REFERENCES}

1. Deplano, A.; Vaneechoutte, M.; Verschraegen, G.; Struelens, M. (1997) Typing of Staphylococcus aureus and Staphylococcus epidermidis Strains by PCR Analysis of Inter-IS256 Spacer Length Polymorphisms. J Clin Microbiol. 35 (10), 2580 - 2587.

2. Hiramatsu, K.; Longzhu, C.; Kuroda, M.; Ito, T. (2001) The emergence and evolution of methicillin-resistant Staphylococcus aureus. Trends in Microbiology. 9 (10), 486 - 493.

3. Huygens, F.; Nimmo, G.; Schooneveldt, J.; Munckhof, W.; Giffard, P. (2002) Genotyping of Methicillin-Resistant Staphylococcus aureus by Assaying for the Presence of Variable Elements Associated with mecA. J Clin Microbiol. 40 (8), 3093-3097.

4. Ito, T.; Katayama, Y.; Asada, K.; Mori, N.; Tsutsumimoto, K.; Tiensasitorn, C.; Hiramatsu, K. (2001). Structural comparison of three types of staphylococcal cassette chromosome mec integrated in the chromosome in methicillin-resistant Staphylococcus aureus. Antimicrob Agents Chemother. 45 (5), 1323 - 1336.

5. Ito, T.; Katayama, Y.; Hiramatsu, K. (1999). Cloning and nucleotide sequence determination of the entire mecA of pre-methicillinresistant Staphylococcus aureus N315. Antimicrob Agents Chemother. 43 (6), 1449 - 1458.

6. Ito, T.; Ma, X.; Takeuchi, F.; Okuma, K.; Yuzawa, H.; Hiramatsu, K. (2004). Novel type staphylococcal cassette chromosome mec driven by a novel cassette chromosome recombinase, ccrC. Antimicrob Agents Chemother. 48 (7), 2637 - 2651.

7. Ito, T.; Okuma, K.; Ma, X.; Yuzawa, H.; Hiramatsu, K. (2003). Insights on antibiotic resistance of Staphylococcus aureus from its whole genome: genomic island SCC. Drug Resist Updat. 6 (1), 41 - 52.

8. Jevons, M. (1961). “Celbenin”-resistant staphylococci. Br Med J. 1: 124125.

9. Milheiriço, C.; Oliveira, D.; Lancastre, H. (2007). Mutiplex PCR strategy for subtyping the staphylococcal cassette chromosome mec type IV in methicillin- resistant $S$. aureus: "SCCmec IV multiplex". J Antimicrob Chemother. 60 (1), 42 - 48.

10. Oliveira, D.; Lencastre.; H. (2002). Multiplex PCR strategy for rapid identification of structural types and variants of the mec element in methicillin-resistant Staphylococcus aureus. Antimicrob Agents Chemother. 46 (7), $2155-2161$.

11. Oliveira, D.; Wu, S.; Lencastre, H. (2000). Genetic organization of the downstream region of the mecA element in methicillin-resistant $S$. aureus isolates carrying different polymorphisms of this region. Antimicrob Agents Chemother. 44 (7), $1906-1910$.

12. Pournaras, S.; Slavakis, A., Polyzou, A.; Sofianou, D.; Maniatis, A.; Tsakris, A. (2001). Nosocomial Spread of an Unusual MethicillinResistant Staphylococcus aureus Clone That Is Sensitive to All 
Non-b-Lactam Antibiotics, Including Tobramycin. J Clin Microbiol. 39 (2), $779-781$.

13. Ryffel, C.; Bucher, R.; Kayser, F.; Berger-Bachi, B, (1991). The Staphylococcus aureus mec Determinant Comprises an Unusual Cluster of Direct Repeats and Codes for a Gene Product Similar to the Escherichia coli sn-Glycerophosphoryl Diester Phosphodiesterase. J Bacteriol. 173 (23), 7416 - 7422.

14. Schmitz, F.; Steiert, M.; Tichy, H.; Hofmann, B.; Verhoef, J.; Heinz, H.; Köhrer, K.; Jones, M. (1998). Typing of methicillin-resistant Staphylococcus aureus isolates from Düsseldorf by six genotypic methods. J Med Microbiol. 47, 341 - 351.

15. Wilson, M.; Otth, C.; Medina, G.; Otth, L.; Fernández, J.; Arce, M.;
Zaror, A.; Lizama, V.; Gil, M.; von Chrismar, A. (2007) Genotipos de Staphylococcus aureus con fenotipo meticilino resistente, aislados de pacientes del Hospital Base de Valdivia. Rev Méd Chile. 135: 596601.

16. Yinduo, Ji. (2007). Methicillin-Resistant Staphylococcus aureus (MRSA) Protocols. Department of veterinary and biomedical sciences, University of Minnesota, St. Paul, MN.

17. Zhang, K.; McClure, J.; Elsayed, S.; Louie, T.; Conly, J. (2005) Novel Multiplex PCR Assay for Characterization and Concomitant Subtyping of Staphylococcal Cassette Chromosome mec Types I to V in Methicillin-Resistant Staphylococcus aureus. J Clin Microb. 43 (10), 5026-5033. 